\title{
Remote login based traffic Performance Analysis of Reactive Routing Protocols of MANETs using OPNET
}

\author{
Gurbhej Singh $^{1}$, Gurjeet Singh ${ }^{2}$ \\ Assistant Professor, Department of Computer Science \& Engineering, SBSSTC, Ferozepur, India ${ }^{1,2}$
}

\begin{abstract}
In MANET routes between nodes changes regularly due mobile nature of all network nodes. It is always challenging task to discover an optimal path. Due to change in network topology some parameters are also effected like network delay, packet drop and throughput etc. There are basically three categories of protocols are reactive, proactive and hybrid. But reactive protocols have wide popularity in this field and provide solution to overcome problems (change in parameters) related to adhoc network. This paper evaluates behaviour of reactive protocols (AODV, DSR) using remote login type traffic in MANET. In this paper some parameters like network delay, total packet drop, network load and throughput are calculated with help of OPNET Modeller 14.5.
\end{abstract}

Keywords: OPNET, MANET, AODV, DSR and Remote login

\section{INTRODUCTION}

MANET is mobile Adhoc network[1,5]. MANET is selforganising group of mobile users which interact with each other with the help of wireless network. MANET consists of number of mobile hosts such as laptops, tablets or mobile with radio interface between them. The interface between hosts is not permanent. The route between them is dynamic that means it change the topology every time, while making connection with other nodes. In adhoc network nodes also act as router that transmit and receive data from other nodes in network. Routing between nodes is very critical issue and there is need to evaluate protocol to make a network reliable.

In adhoc network there are two basic routing techniques. First one is proactive and second is reactive routing. Proactive routing is based on the formula that routing protocol makes decision to get router, when there is need to transmit packet. In proactive protocol routes of packet changes very quickly before reaches to destination due to change in topology. DSDV and WRP protocol are example of proactive routing. Reactive routing selects route according to the requirement. When there is a packet to transmit, protocol selects routes a suitable route from source to destination nodes and this route remain unchanged before reaching the destination node. AODV, DSR are example or reactive protocol.

\section{REACTIVE ROUTING PROTOCOLS}

2.1 Dynamic source routing protocol (DSR)- DSR is reactive routing protocol. $[8,10]$ This protocol is purely on-demand routing protocol. Every node has cache memory which is used to stored possible paths between source and destination. In DSR node can store more than one route in cache memory. Source node can check and validate route before starting the transmition. DSR updates cache of node by discovering new routes. The new routes are added in cache if there exist a direct path from source to destination node. When source node has data to transmit over the network towards destination then it defines route.

The transmitting data will follow same route in network. There is no need of 'hello message' exchange between nodes so nodes remains in sleep mode and consume very less energy. In DSR small portion of bandwidth is used. In this protocol source node multicast routing request (RREQ). This request includes source identification (SID), destination identification (DID), and unique request sequence number called Request ID (RID). a). Routing Discovery Process in DSR: This mechanism is used to discover path from source to destination. b). Route Maintenance in DSR: This mechanism take cares of connection after transmition start. Maintenance checks lost of packet and error in data etc

2.2 Adhoc On-demand Vector Routing protocol (AODV)- AODV is also a reactive protocol. AODV use control message to discover route from source to destination before starting transmition. In AODV source node sends request message to all its neighbours. This process is followed until destination is reached or time expired. If destination reached then destination node make reply to source node. In this process route discovered and route is used to transmit data.[7,9]

\section{III.SIMULATION SETUP}

In this paper our motivation is to analyze behaviour of reactive routing protocols (AODV, DSR).For evalution of AODV and DSR OPNET 14.5 Simulator[6,10] is used. The network area is taken $2000 \mathrm{~m} * 2000 \mathrm{~m}$. The mobile nodes are spread over this area of interest or network area. The numbers of mobile nodes are different for two cases. In first case numbers of mobile nodes are 25 and 50 for second case. The time for simulation is taken as 600 seconds and traffic used to simulation is remote login. Remote login application and profile is configured in this paper. The network is wireless LAN for mobile nodes having a data rate of $11 \mathrm{Mbps}$ and random waypoint mobility model used in this simulation. DES's (global discrete event statistics) are collected on both protocols 
and wireless LAN. The mobility model used in this 3.1.1 Delay

simulation is simple and it shows good mobility The Time of generation of a packet by the source up to the behaviour. This simulation is used to check behaviour of destination reception is end to end delay. So delay is time reacting routing protocol AODV and DSR. Figure 3.1 taken by packet to go across the network.Delay is and3.2 shows simulation setup for 25 and 50 mobile node expressed in seconds. Processing delay (PD), queuing respectively. The important parameters used to evaluate delay (QD), transmission delay (TD) and propagation performance of both protocols are delay, total packet lose, delay (PD)are called packet end to end delay in network. network load and throughput

Table 1 Simulation Parameter

\begin{tabular}{|c|c|}
\hline Routing protocol & AODV, DSR \\
\hline MAC layer & $802.11(\mathrm{DCF})$ \\
\hline MANET size & $2000 \mathrm{~m}$ X 2000 m \\
\hline Mobile nodes & 25,50 \\
\hline Mobility models & Random waypoint mobility \\
\hline Traffic type & Remote login(High Speed) \\
\hline Simulation time & 600 Seconds \\
\hline PHY standard used & $802.11 \mathrm{~b}$ \\
\hline Data Rate & $11 \mathrm{Mbps}$ \\
\hline
\end{tabular}

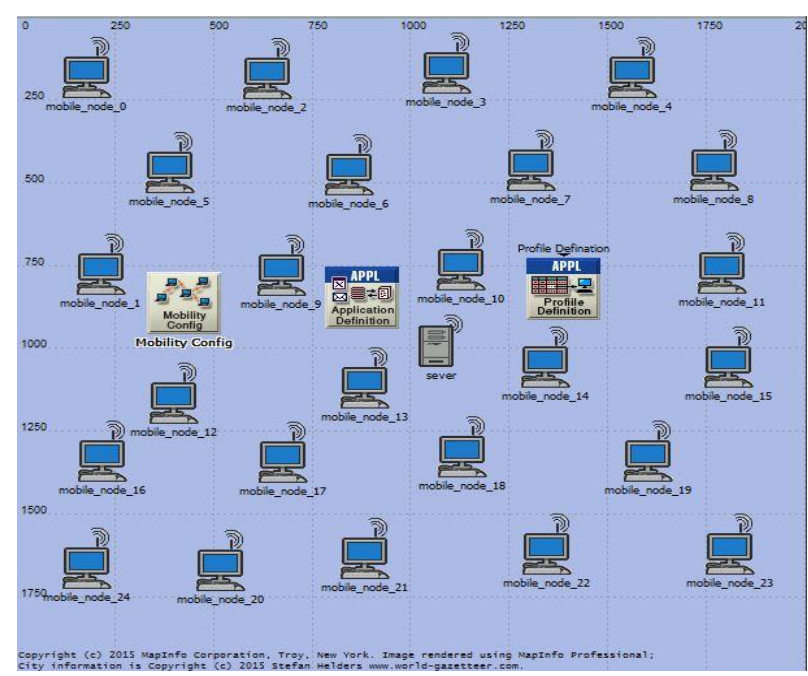

Figure 3.1(a) Model of WLAN network using OPNET simulator

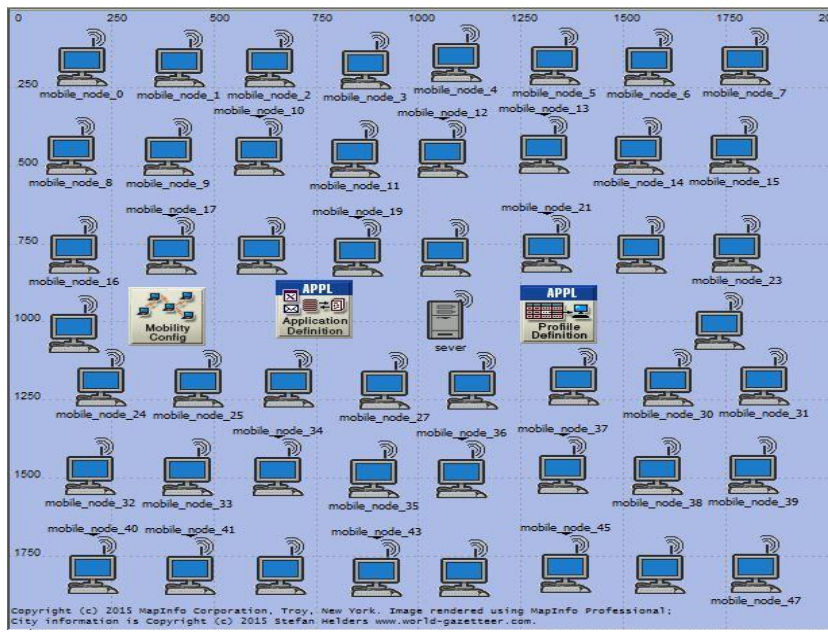

Figure 3.1(b)

\subsection{Performance parameters}

The Remote Login traffic is considered here for the simulations since it is applicable in internet applications.
Latency time and delay has same meaning.

\subsubsection{Network Load}

Network load represents the total load in bit per second submitted to wireless LAN layers by all higher layers in all WLAN nodes of the network is called Network Load. [3]

\subsubsection{Throughput}

Throughput is ratio of the total data reaches a receiver from the sender. The time it takes by the receiver to receive the last message is called as throughput [2]

Topology changes in the network, unreliable communication between nodes, limited bandwidth available and limited energy are the factors that affects the throughput.Throughput is expressed as bytes or bits per sec (byte/sec or bit/sec). Throughput can be defined by the following formula [4]:

Throughput $=$ (number of delivered packet $*$ packet size)/total duration of simulation

\section{IV.ANALYSIS AND RESULTS}

The reactive protocols (AODV, DSR) are evaluated under different circumstances to check their efficiency in network. Results and discussion part includes performance checking parameters (delay, total packet drop, network load and throughput) and some other factors that can effect performance.

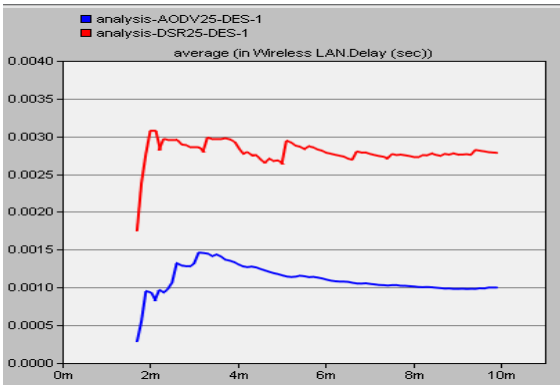

Figure 4.1 Measurement of delay in 25 nodes using different reactive adhoc routing schemes

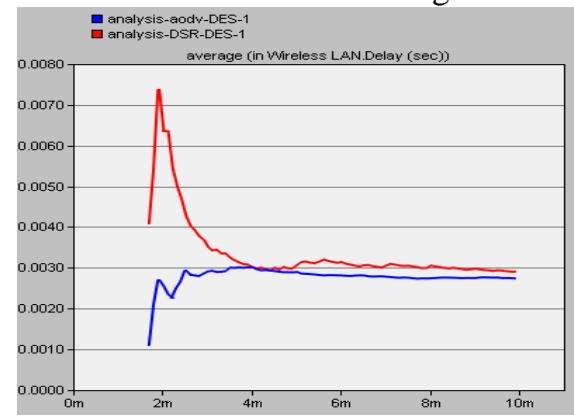

Figure 4.2 Measurement of delay in 50 nodes using different reactive adhoc routing schemes

Figure 4.1 represents delay for both AODV and DSR when there are 25 mobile nodes in network of $2000 \mathrm{~m} *$ $2000 \mathrm{~m}$. This graph shows DSR having more delay as 
compare to AODV. In both techniques initially delay increases sharply and becomes almost constant. AODV have average delay for whole simulation time .0010 and 0.0027 and figure 4.2 represents delay for same protocols when they have 50 mobile nodes over network. When network have 50 mobile nodes then average delay for DSR is nearly .0038 and .0027 for AODV. In Figure 4.1 and 4.2 red and blue lines represents delay of protocols AODV and DSR respectively.

Figure 4.3 and 4.4 represents total packet drop for both AODV and DSR when there are 25 and 50 mobile nodes respectively in network of $2000 \mathrm{~m} * 2000 \mathrm{~m}$.AODV having more packet drop as compare to DSR.DSR protocol have very low and constant packet drop of 1 in both cases having nodes 25 and 50 due to good path selection technique. AODV protocol have variable packet drop from 2 to 10 but average value nearly 7 but at in second case with 50 nodes packet drop sharply increases from 1 to 12 and average packet drop nearly 9.5. In figure 4.3 and figure 4.4 red and blue lines represents total packet drop for protocols DSR and AODV respectively.

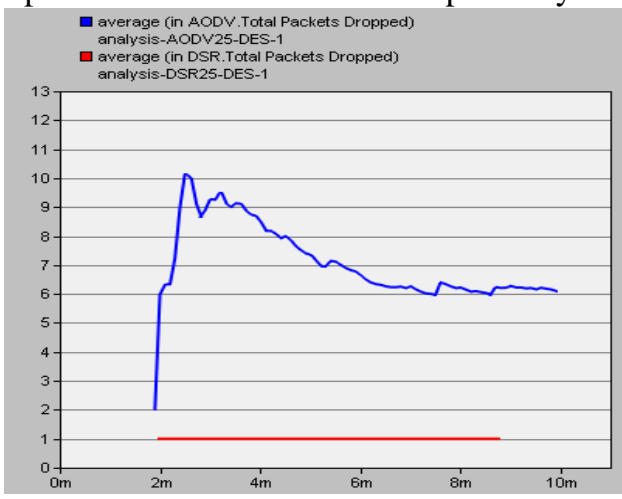

Figure 4.3 Measurement of Total Packet Drop in 25 nodes using different reactive adhoc routing schemes

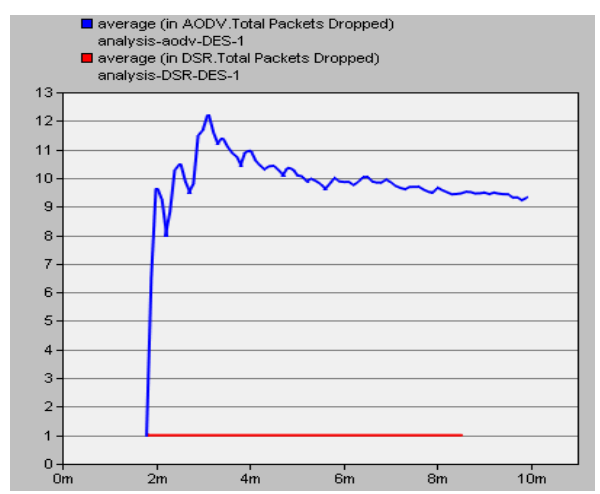

Figure 4.4 Measurement of Total Packet Drop in 25 nodes using different reactive adhoc routing schemes

Figure 4.5 represents network load for both AODV and DSR when there are 25 and 50 mobile nodes. In graph (4.5) red and blue lines represents network load for AODV having 25 and 50 nodes respectively. Black and parrot colour lines represents network load for DSR protocol having nodes 50 and 25 respectively.

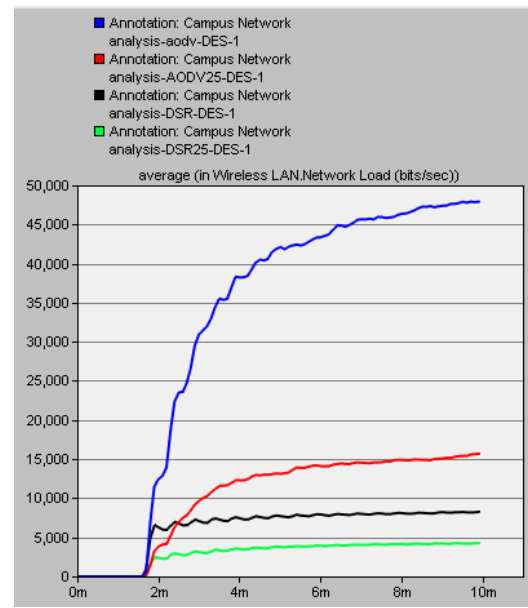

Figure 4.5 Measurement of Network Load in 25 and 50 nodes using different reactive adhoc routing schemes

Figure 4.5 represents throughput for both AODV and DSR when there are 25 and 50 mobile nodes. In figure 4.6 red and blue lines represents throughput for AODV having 25 and 50 nodes respectively. Black and parrot colour lines represents network load for DSR protocol having nodes 50 and 25 respectively.

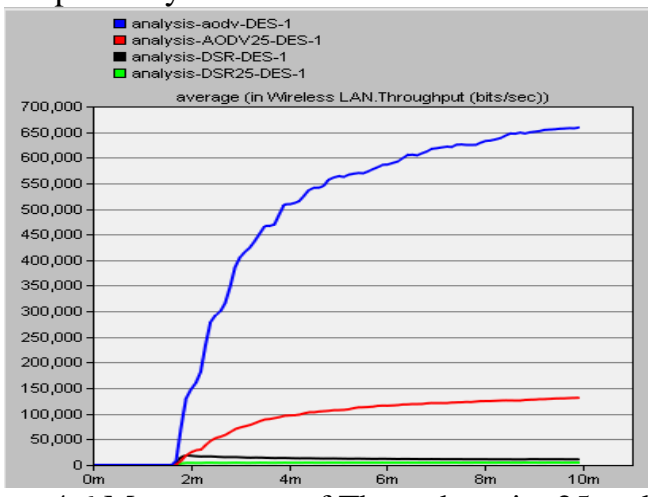

Figure 4.6 Measurement of Throughput in 25 and 50 nodes using different reactive adhoc routing schemes

\section{Conclusion}

In this paper two reactive protocols(AODV and DSR) are anaylsed for 25 and 50 nodes for remote login trafic in MANET. It includes four parmeters(throughput, total packet drop, delay and network load) for comparison between two protocols. It is observed from simulation AODV has more throrghput as compare to DSR, and AODV gives higher throughput at 50 as compare to 25 nodes in netwrk area $2 \mathrm{~km} * 2 \mathrm{~km}$. DSR have less total packet drop and network load as compare to AODV. DSR have high delay in both cases(for 25,50 nodes) as compare to AODV.AODV is best suited routing protocol in dence population of nodes for MANET with remote login trafffic.

\section{ACKNOWLEDGMENT}

The authors wish to thank Mr. Sikandar Singh Cheema, Assistant Professor (Computer Science and Engineering), Punjabi University ,Patiala for his guidance and support in research. 


\section{REFERENCES}

[1] David B. Johnson and David A.Maltz, "Dynamic source routing in ad hoc wireless networks". In Mobile Computing, edited by Tomasz Imielinski and Hank Korth, chapter 5, pages 153-181. Kluwer Academic Publishers.

[2] Uyen Trang Nguyen and Xing Xiong, "Rate-adaptive Multicast in Mobile Ad-hoc Networks', Department of Computer Science and Engineering York University Toronto, Canada M3J 1P3.

[3] Suhaimi Bin Abd Latif, M.A. Rashid, F.Alam, "Profiling Delay and Throughput Characteristics of Interactive Multimedia Traffic over WLANs using OPNET', School of Engineering Institute of Technology \& Engineering, Massey University, Auckland, New Zealand

[4] Parulpreet Singh, Ekta Barkhodia, Gurleen Kaur Walia ,'Evaluation of various Traffic loads in MANET with DSR routing protocol through use of OPNET Simulator", International Journal of Distributed and Parallel Systems (IJDPS) Vol.3, No.3, May 2012 DOI : $10.5121 /$ ijdps.330875,2012

[5] G. Pei, M. Gerla and T.-W. Chen, (Apr. 2000, pp. D71-D78) Fisheye State Routing in Mobile Ad Hoc Networks. In Proceedings of the 2000 ICDCS Workshops, Taipei,Taiwan

[6] Opnet Technologies, Inc. "Opnet Simulator," Internet: www.opnet.com, date last viewed: 2014-12-12

[7] C. Perkins, E.M. Royer, S.R. Das, Ad Hoc on Demand Distance Vector (AODV) Routing Internet Draft, IETF, 1999.

[8] J. Broch, D.B. Johnson, D.A. Maltz, The dynamic source routing in ad hoc wireless networks, in: T. Imielinski, H. Korth (Eds.), Mobile Computing, Kluwer Academic Publishers, Dordrecht, 1996, pp. 153-181.

[9] M. Abolhasan, T. Wysocki, E. Dutkiewic, A review of routing protocols for mobile ad hoc networks Adhoc Networks, vol. 2, Elsevier, 2004, pp. 1-22.

[10] Pankaj Palta and Sonia Goyal , "Comparison of OLSR and TORA routing protocols using OPNET Modeler" in International Journal of Engineering Research and technology Vol. 1 Issue 5, July - 2012

\section{BIOGRAPHIES}

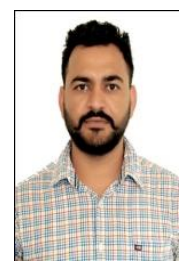

Gurbhej Singh has received his Master of Technology Degree in Computer Science and Engineering from Sri Guru Granth Sahib World University, Fatehgarh Sahib. $\mathrm{He}$ has received his B.Tech degree from Punjab Technical University, Jalandhar, India. Presently he is working as Assistant Professor in Department of Computer Science and Engineering at Shaheed Bhagat Singh State Technical Campus, Ferozepur. His research interest is Computer Networks.

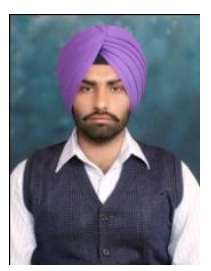

Gurjeet Singh has received his Master of Technology Degree in Computer Science and Engineering from Punjab Agriculture University, Ludhiana. He has received his B.Tech degree from Punjab Technical University, Jalandhar, India. Presently he is working as Assistant Professor in Department of Computer Science and Engineering at Shaheed Bhagat Singh State Technical Campus, Ferozepur. His research interest is Computer Networks. 